\title{
e Intrathecal Bupivacaine Monotherapy with a Retrograde Catheter for the Management of Complex Regional Pain Syndrome of the Lower Extremity
}

\author{
W. Porter McRoberts, MD', Catalina Apostol, MD², and Abdul Haleem, MBBS
}

From: ${ }^{1}$ Holy Cross Hospital, Dept. of Pain Medicine, Fort Lauderdale, FL; ${ }^{2}$ Holy Cross Orthopedic Institute, Dept. of Pain Medicine, Fort Lauderdale, FL

Address Correspondence: W. Porter McRoberts, MD Holy Cross Hospital Dept. of Pain Medicine 5601 North Dixie Highway

Fort Lauderdale, FL 33334 E-mail: portermcroberts@gmail.com

Disclaimer: There was no external funding in the preparation of this manuscript.

Conflict of interest: Each author certifies that he or she, or a member

of his or her immediate family, has

no commercial association (i.e. consultancies, stock ownership, equity interest, patent/licensing arrangements, etc.) that might pose a conflict of interest in connection with the submitted manuscript.

Manuscript received: 01-04-2016 Accepted for publication: 02-03-2016

Free full manuscript: www.painphysicianjournal.com
Complex regional pain syndrome (CRPS) presents a therapeutic challenge due to its many presentations and multifaceted pathophysiology. There is no approved treatment algorithm and clinical interventions are often applied empirically. In cases of CRPS where symptoms are localized to an extremity, a targeted treatment is indicated. We describe the use of intrathecal bupivacaine monotherapy, delivered through a retrograde catheter, in the treatment of CRPS affecting the lower extremity. The patient, a 57-yearold woman with a history of failed foot surgery, was seen in our office after 2 years of ineffective treatments with local blocks and neurolytic procedures. We advanced therapy to moderately invasive procedures with an emphasis on neuromodulation. A combined central and peripheral stimulation technique that initially provided $75 \%$ pain relief, failed to provide lasting analgesia. We proceeded with an intrathecal pump implant. Based on the results of dorsal root ganglion (DRG) mapping, L5-S1 was identified as the optimal target for therapy and a retrograde catheter was placed at this level. Various intrathecal medications were tested individually. An intrathecal morphine trial was ineffective (visual analog scale [VAS] 7), while intrathecal clonidine provided excellent pain relief (VAS 0 ) that was limited by severe side effects. Bupivacaine provided $100 \%$ analgesia with tolerable side effects (lower extremity weakness and minor bladder incontinence) and was selected for intrathecal infusion. After 14 months, bupivacaine treatment continued to control pain exacerbations. We conclude that CRPS patients benefit from early identification of the predominant underlying symptoms and a targeted treatment with moderately invasive techniques when less invasive techniques fail.

Key words: Intrathecal bupivacaine, bupivacaine monotherapy, retrograde catheter, complex regional pain syndrome (CRPS), dual stimulation, dosal root ganglion (DRG) testing

Pain Physician 2016; 19:E1087-E1092

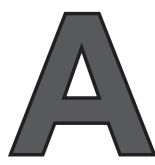
ccording to the Polyanalgesic Consensus Conference guidelines, targeted intrathecal (IT) therapy is recommended for the treatment of chronic non-cancer pain when less invasive therapies fail (1). Direct analgesic delivery to the neural axis offers immediate access to receptors, bypasses the blood-brain barrier, and minimizes systemic drug interactions (2). A commonly used mixture for the treatment of intractable pain consists of morphine and bupivacaine (3). According to a retrospective study of non-cancer patients displaying opioid resistance after long-term IT infusions, addition of bupivacaine significantly lowered visual analog scale (VAS) scores and improved the quality of life. The addition of IT bupivacaine, however, provided better analgesia in patients with neuropathic pain than in patients with nociceptive pain $(4,5)$. Some studies suggest that opioids lack a significant analgesic effect on neuropathic and idiopathic forms of pain (6) and recommend performing an initial opioid test 
to determine whether opioids should be part of the treatment plan. Opioid complications may outweigh the benefits especially in younger, non-cancer patients who need to be functional (7). Risks associated with IT opioids (respiratory depression, peripheral edema, granuloma formation) (1) are eliminated by judiciously identifying the mechanisms of pain and guiding treatment accordingly. In patients with predominantly neurogenic pain, bupivacaine monotherapy can provide a safer and more effective IT therapy than an admixture of bupivacaine and opioids.

Although IT bupivacaine is not FDA approved, multiple studies support its long-term safety in the treatment of chronic pain. A 2002 MEDLINE overview of studies employing IT bupivacaine, found this treatment acceptable for chronic pain in cancer and non-cancer patients (8). According to a 1998 prospective, cohort study, IT bupivacaine provided better pain relief than epidural dosing and toxicity was not seen at clinically relevant doses (9). IT bupivacaine was compatible with pump materials and its concentration remained stable when stored in a SynchroMed pump (Medtronic, Inc., Minneapolis, MN, USA) over 12 weeks (10). A targeted bupivacaine therapy is indicated for the treatment of complex regional pain syndrome (CRPS) patients with predominant sensory abnormalities and localized extremity pain. We present a case of intractable foot pain in a patient with CRPS II, where IT bupivacaine monotherapy provided the best analgesia with the fewest complications. We administered bupivacaine through a retrograde IT catheter, targeting the nerve roots that innervated the affected extremity.

\section{Case Study}

A 57-year-old woman presented to our clinic with a 2 year history of right foot pain, resulting from nerve and fascial injury following a failed plantar fasciitis procedure. The pain covered the medial and plantar aspect of the foot, and was exacerbated by walking. There was no improvement with physical therapy or pharmacotherapy (oxycodone, lidocaine patches, tramadol, escitalopram, bupropion, and oral steroids). In the first month after surgery, the patient also underwent a calcaneal neurectomy and tarsal tunnel decompression without improvement.

In the following year, the patient consulted multiple specialists. Thorough investigation showed no evidence of nerve entrapment, infection, or malignancy. Electromyogram results suggested a possible right medial plantar nerve injury. Magnetic resonance imaging further indicated a right plantar fascia defect, likely a result of surgery. The right foot was immobilized with a cast to allow healing while the patient, now wheelchair-bound, continued treatment with minimally invasive procedures. A series of blocks provided insignificant relief: a lumbar sympathetic block, ankle nerve blocks, steroid injections, and a lumbar epidural at the L4-L5 and L5-S1 levels. Resection of the medial calcaneal branch of the posterior tibial nerve resulted in numbness without improvement in pain. A series of 8 alcohol injections provided adequate analgesia and permitted ambulation. However, pedal edema developed and the pain returned after 2 months.

The patient presented to our clinic with a 24 month history of right lower extremity pain. On exam, she had edema, reduced range of motion, allodynia, and skin color changes in the right foot (Fig. 1). After a positive lumbar sympathetic block (VAS decreased from 7/10 to 2/10 over a period of 5 days), we diagnosed the patient with CRPS type II. In addition to sympathetically mediated pain, we identified a predominant neuropathic component and advanced treatment to central stimulation. A trial at the T9 level had favorable results: VAS decreased to 2/10, activity increased, and oral opioids were discontinued.

While awaiting stimulator implant authorization, the patient also underwent dorsal root ganglion (DRG) mapping, with the expectation that if the pain improved significantly, she could receive a DRG stimulator, once approved by the FDA. DRG mapping was performed via a transforaminal epidural approach, using a shielded radiofrequency 22 gauge needle. There was a positive response at the L5 and S1 levels and a negative response (no pain relief) at L4 and S2. Unfortunately, the DRG stimulation device required a waiting period that was unacceptable to the patient given her current level of pain. We proceeded with permanent implant of a spinal cord stimulator and supplemented it with a peripheral lead to the posterior tibial nerve (Fig. 2).

After one month of central and peripheral stimulation, the patient reported good pain relief (VAS 4/10) and a reduction in exacerbations from once a day to just once a month. Five months post implant, the pain returned with such severity (VAS 8/10) that the patient requested foot amputation. She was seen in the emergency room on several occasions and treated with hydromorphone drip. Ketamine infusion was attempted in another pain clinic and aborted due to dissociative symptoms. In our office, we performed a posterior tibial nerve block that gave $60-70 \%$ pain relief. We sus- 


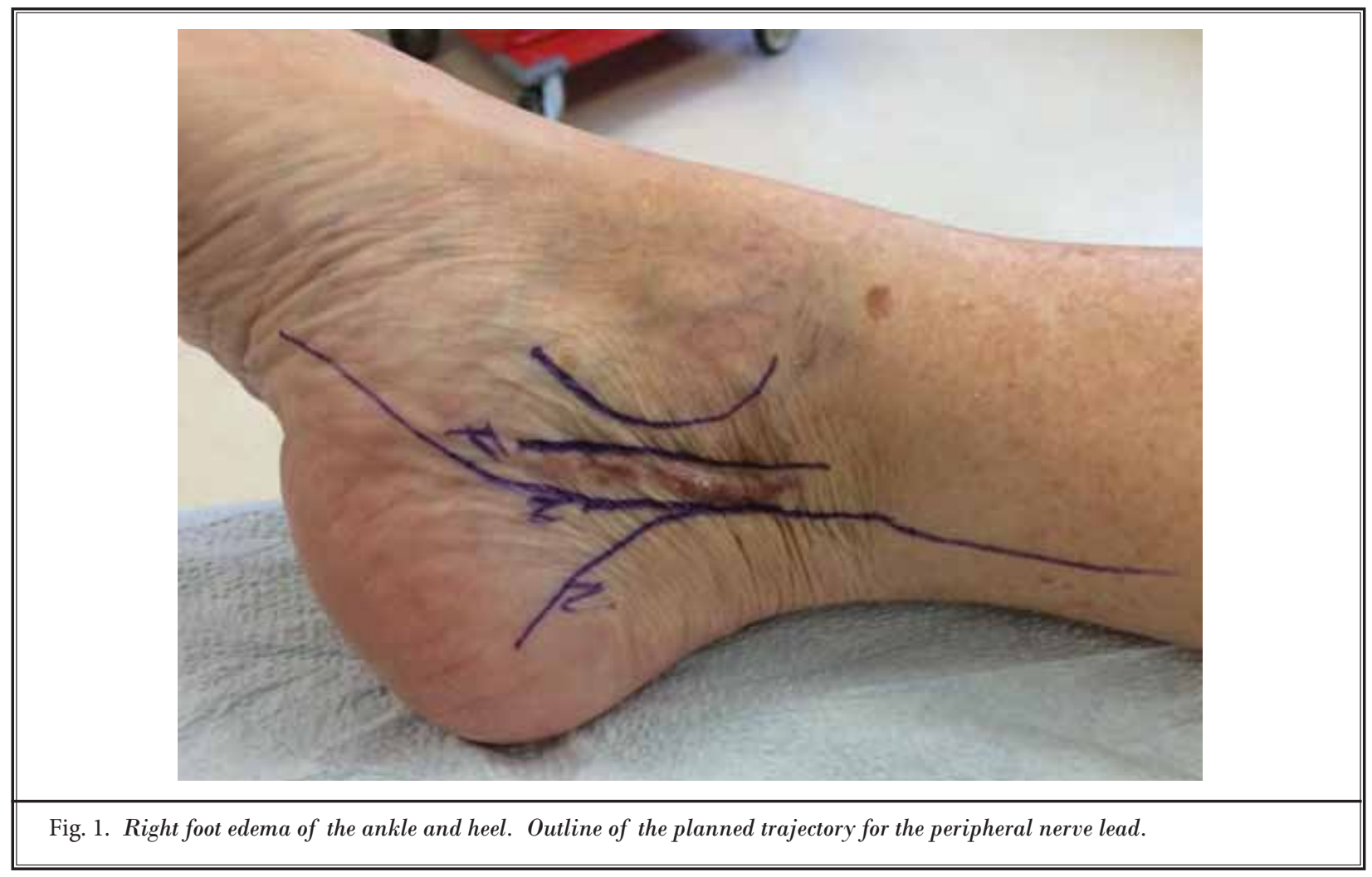

pected overstimulation and advised the patient to use her stimulator intermittently.

Six months after implant, stimulation was no longer offering significant pain relief. We decided to add an IT pump and performed IT trials with morphine and, separately, with lidocaine. Morphine offered minimal relief (VAS 7). Lidocaine decreased the pain to a VAS level of zero while producing a motor block of the lower extremities. In order to avoid this limitation, we proceeded with a clonidine trial. Based on a favorable trial (VAS 0), a Medtronic $20 \mathrm{~mL}$ SynchroMed II programmable pump (Medtronic, Inc., Minneapolis, MN, USA) was implanted for delivery of a continuous clonidine infusion. The IT catheter was placed in a retrograde fashion, with the tip at the L5-S1 interspace, right of the midline, in the lateral recess (Fig. 3). A myelogram with contrast solution showed excellent local spread. The patient had good pain relief (VAS 2/10) with clonidine for several days until she started experiencing headaches, night sweats, weakness, and a low blood pressure requiring treatment in the emergency room. Clonidine was discontinued.

A bupivacaine trial was then attempted and gave $100 \%$ pain relief. Therapy was switched to a hypobaric

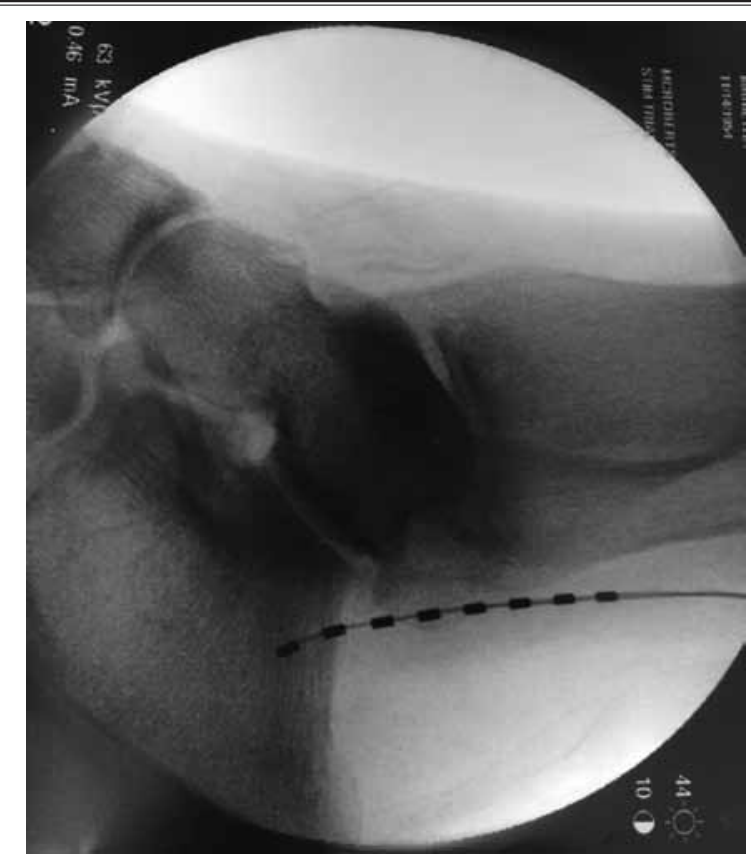

Fig. 2. Radiographic image of the peripheral 8 contact coaxial lead, inserted along the trajectory of the right posterior tibial nerve. 


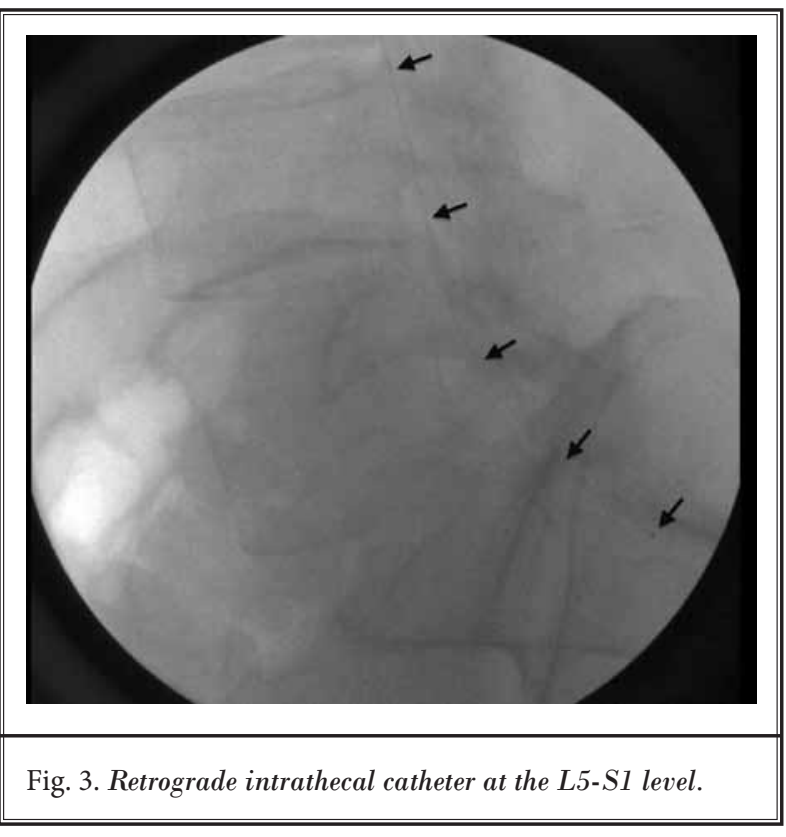

bupivacaine infusion (Medisca Inc., Plattsburgh, NY, USA): $3 \mathrm{mg} /$ day with $2-4 \mathrm{mg}$ boluses up to 10 times per day. After multiple dose adjustments meant to decrease a bupivacaine-related perineal anesthesia and extremity motor block, the pump was programmed to deliver an infusion of $3 \mathrm{mg} / \mathrm{day}$ and $1.85 \mathrm{mg}$ bupivacaine boluses, up to 10 times a day. The patient experienced $90-$ $95 \%$ relief using a daily average of 4 boluses that lasted between 30 minutes and 4 hours. This enabled her to ambulate for 10 - 15 minutes at a time and perform chores without her electrical scooter. We helped her experiment with positioning during boluses, and found that a left lateral decubitus position, with the right foot elevated, minimized the unwanted sensory blockade in the opposite foot and perineum. Although pain still peaked between boluses (VAS 8), the patient discontinued narcotics and considered her current pain level a significant improvement over her initial condition.

\section{Discussion}

CRPS presents a therapeutic challenge due to its many presentations and multifaceted pathophysiology. Although we know that both peripheral and central mechanisms are involved, a better understanding of the underlying pathology is needed. There is no approved treatment algorithm and clinical interventions are often applied empirically, to counter the various symptoms as they arise (11). As part of our treatment, we tried several invasive modalities, starting with stim- ulation and later advancing to an IT pump. An ideal starting point in our investigations was finding the anatomic source for the pain. DRG mapping was essential in identifying the L5 and S1 levels as the best targets for therapy. Based on this information, we threaded a retrograde catheter to these levels of the spinal nerve roots. In order to maximize analgesia and avoid undesirable side effects, we decided to test various IT drugs, sequentially. We started with an initial morphine trial that was negative, thus allowing us to eliminate opioids from the treatment algorithm. Continued testing, showed that clonidine and bupivacaine provided the best pain control. Clonidine had severe side effects, leaving bupivacaine as the preferred agent. A dual targeted approach involving a retrograde catheter and bupivacaine IT monotherapy gave the best results in our patient.

CRPS presents an obvious therapeutic challenge. We believe, both central and peripheral mechanisms are involved. However symptoms vary and there is no approved treatment algorithm, to date. According to Stanton-Hicks et al (12) interventional treatment can be generally divided in 3 stages: minimally invasive therapies (blocks), more invasive therapies (stimulators, IT pumps), and surgical ablations (sympathectomy) as a last resort. Secondary to the poorly understood nature of CRPS and lack of clinical consensus, a variety of specialties may be involved in the treatment. Neurodestructive procedures (neurectomy, sympathetic ablation, and neurolytic alcohol blocks) were performed in the early stages of treatment, and likely exacerbated our patient's situation by inducing post-sympathectomy neuralgia. According to the latest Practical Diagnostic and Treatment Guidelines for CRPS, neuro-destructive procedures are best relegated to terminally ill patients (11). We believe that non neuro-destructive second stage interventions should be the main treatment for CRPS and that the pain practitioner should be at the center of treatment planning.

IT bupivacaine monotherapy is infrequently used for CRPS treatment. A 1999 case study by Lundberg and colleagues (13) examined the effects of continuous IT bupivacaine infusion in 2 patients with CRPS-I. The authors concluded that this treatment provided some pain relief but did not stop the associated symptoms of edema from spreading to the entire extremity or the opposite limb. They did not recommended IT bupivacaine in preference to other pain treatment regimens for CRPS-I. Our findings, however, support the use of IT bupivacaine monotherapy for CRPS treatment when 
symptoms are predominantly localized sensory abnormalities and neuropathic pain. In these patients, IT local anesthetics may be effective due to the blocking of sodium channels in neuron membranes and the interruption of impulses associated with neuropathic and inflammatory pain. The neuropathic pain subgroup is different from other subgroups who exhibit predominant vasomotor signs or severe reflex sympathetic dystrophy findings (muscle atrophy, tendon contracture, and osteoporosis). The Fourth Edition of the CRPS Practical Diagnostic and Treatment Guidelines (11) suggests that labeling patients according to their primary symptoms allows the identification of a target treatment more effectively (14) than the historical chronological description of acute, dystrophic, and atrophic stages (15).

A negative IT opioid test guided our therapy towards infusion with clonidine and later with bupivacaine. Clonidine, although an effective analgesic, was limited by its severe side effects. Retrospectively, we believe that better coordination between the pain management team and the patient's cardiologist, with careful weaning from existing oral hypertensives as the patient started IT clonidine, might have prevented the adverse effects that led to the exclusion of clonidine. Although effective, a limitation of using bupivacaine was the unwanted motor block in the opposite extremity and the minor bladder incontinence. Various dose changes were made and the patient was advised to bolus herself in a right lateral position, in order to minimize the unwanted sensory and motor block. Another limitation of our treatment was the absence of a method for prolonged pain relief. IT bupivacaine boluses were effective for pain exacerbations but lacked an extended analgesic effect. We initially planned to use central and peripheral stimulation as a modality of sustained pain relief. Combined central-peripheral stimulation improved VAS scores by $75 \%$ during the trial and $60 \%$ one month after implant. Unfortunately, analgesia progressively decreased over the course of 6 months and we proceeded with the IT pump. DRG stimulation holds potential for sustained analgesia and may offer future direction for the treatment of sympathetically mediated and neuropathic pain. Pending its approval by the FDA, we plan to offer it as a sustained treatment modality to our patient.

Formal cohort prospective studies would be beneficial in finding more about the cost and outcome of IT bupivacaine as a customized therapy for CRPS. A better understanding of the CRPS mechanism(s) may lead to an improved treatment algorithm that specifies when to proceed from minimally invasive techniques to more invasive therapies. Timely therapy is essential in CRPS. Symptoms can extend in the affected extremity and even spread to the contralateral one, leading to infections and possible amputation (13). Based on our experience, we recommend classifying CRPS patients according to symptoms, and customizing the treatment. For patients with predominant neuropathic pain, we recommend doing an initial IT opioid test which might exclude opioids from therapy. An often overlooked contraindication of long-term IT opioid infusions is extremity edema. Over a period of 24 months, IT opioids were found to exacerbate preexisting pedal edema and prevent physical activity in 5 out of 23 non-cancer patients (16). Edema compromises tissue oxygenation in CRPS, leading to ulceration, infection, and possible amputation. We believe that using opioids in patients who are unresponsive to an initial opioid trial, is an unnecessary risk. Monotherapy with bupivacaine or clonidine may be the better alternatives. We also recommend using retrograde IT catheters for lower extremity pain, in order to direct delivery of local anesthetics to the neurologic levels involved in the pain mechanism.

\section{Conclusion}

In conclusion, this case report described a CRPS patient, with neuropathic symptoms, resulting in debilitating right foot pain. She had undergone repeated minimally invasive blocks and several neuro-destructive procedures as part of her initial treatments, and experienced an exacerbation of pain. We subsequently advanced her treatment to more invasive techniques, and found that IT monotherapy with bupivacaine offered the best relief with the least severe side effects. The patient reported $100 \%$ pain relief after treatment with bupivacaine boluses, delivered through a retrograde catheter at the L5-S1 level. Unfortunately, she had some degree of motor block in her left foot and minimal bladder incontinence. Overall, she felt the treatment increased mobility and function. This case illustrates the benefit of characterizing CRPS patients among 3 main classes: sensory abnormalities with limited neuropathic pain, vasomotor abnormalities, or RSD \{sp\}-like symptoms. Treatment can then be targeted to the particular symptoms. Our patient benefited from individualized treatment, with IT monotherapy through a retrograde catheter. We endorse a targeted therapy and practitioner tenacity to seek alternate treatments as symptoms evolve. 


\section{References}

1. Deer TR, Levy R, Prager J, Buchser E, Burton A, Caraway D, Cousins M, De Andrés J, Diwan S, Erdek M, Grigsby E, Huntoon M, Jacobs MS, Kim P, Kumar K, Leong M, Liem L, McDowell II GC, Panchal S, Rauck R, Saulino M, Sitzman TB, Peter S. Polyanalgesic Consensus Conference-2012: Recommendations to reduce morbidity and mortality in intrathecal drug delivery in the treatment of chronic pain. Neuromodulation 2012; 15:467-482.

2. Belverud SA, Mogilner AY, Schulde M. Intrathecal bupivacaine for head and neck pain. Local Reg Anesth 2010; 3:125128.

3. Hassenbusch SJ, Stanton-Hicks M, Covington EC, Walsh JG, Guthrey DS. Longterm intraspinal infusions of opioids in the treatment of neuropathic pain. J Pain Symptom Manage 1995; 10:527-543.

4. Kumar K, Bodani V, Bishop S, BN, Tracey $S$. Use of intrathecal bupivacaine in refractory chronic nonmalignant pain. Pain Med 2009; 10:819-828.

5. Krames ES, Lanning RM. Intrathecal infusional analgesia for nonmalignant pain: analgesic efficacy of intrathecal opioid with or without bupivacaine. J Pain Symptom Manage 1993; 8:539-548.
6. Arner S, Meyerson BA. Lack of analgesic effect of opioids on neuropathic and idiopathic forms of pain. Pain 1988; 33:1123.

7. Buntin-Mushock C, Phillip L, Moriyama K, Palmer PP. Age-dependent opioid escalation in chronic pain patients. Anesth Analg 2005; 100:1740-1745.

8. Deer TR, Serafini M, Buchser E, Ferrante FM, Hassenbusch SJ. Intrathecal bupivacaine for chronic pain: A review of current knowledge. Neuromodulation 2002; 5:196-207.

9. Dahm P, Nitescu P, Appelgren L, Curelaru I. Efficacy and technical complications of long-term continuous intraspinal infusions of opioid and/or bupivacaine in refractory nonmalignant pain: A comparison between the epidural and the intrathecal approach with externalized or implanted catheters and infusion pumps. Clin J Pain 1998; 14:4-16.

10. Hildebrand KR, Elsberry DD, Deer TR. Stability, compatibility, and safety of intrathecal bupivacaine administered chronically via an implantable delivery system. Clin J Pain 2001; 17:39-44.

11. Harden RN, Oaklander AL, Burton AW, Perez RS, Richardson K, Swan M, Barthel
J, Costa B, Graciosa JR, Bruehl S. Complex regional pain syndrome: Practical diagnostic and treatment guidelines, 4th edition. Pain Med 2013; 14:180-229.

12. Stanton-Hicks M, Burton A, Bruehl SP, Carr DB, Harden RN, Hassenbusch S), Lubenow TR, Oakley JC, Racz GB, Raj PP, Rauck RL, Rezai AR. An updated interdisciplinary clinical pathway for CRPS: Report of an expert panel. Pain Pract 2002; 2:1-16.

13. Lundborg C, Dahm P, Nitescu P, Appelgren L, Curelaru I. Clinical experience using intrathecal (IT) bupivacaine infusion in three patients with complex regional pain syndrome type I (CRPS-I). Acta Anaesthesiol Scand 1999; 43:667-678.

14. Veldman $\mathrm{PH}$, Reynen $\mathrm{HM}$, Arntz IE, Goris RJ. Signs and symptoms of reflex sympathetic dystrophy: Prospective study of 829 patients. Lancet 1993; 342:1012-1016.

15. Bonica JJ. The Management of Pain. Lea and Feibiger, Philadelphia, PA, 1953.

16. Aldrete JA, Couto da Silva JM. Leg edema from intrathecal opiate infusions. Eur J Pain 2000; 4:361-365. 